PROCEEDINGS OF THE AMERICAN MATHEMATICAL SOCIETY

Volume 124, Number 10, October 1996

\title{
EQUIVALENT CONDITIONS INVOLVING COMMON FIXED POINTS FOR MAPS ON THE UNIT INTERVAL
}

\author{
JACEK R. JACHYMSKI
}

(Communicated by James E. West)

\begin{abstract}
Let $g$ be a continuous self-map of the unit interval $I$. Equivalent conditions are given to ensure that $g$ has a common fixed point with every continuous map $f: I \mapsto I$ that commutes with $g$ on a suitable subset of $I$. This extends a recent result of Gerald Jungck.
\end{abstract}

\section{INTRODUCTION}

Let $f$ and $g$ be two commuting continuous self-maps of $I$, the unit interval. It is known that $f$ and $g$ need not have then a common fixed point (for counterexamples, see, e.g., [6]). However, if one of the maps, say $g$, has appropriate additional properties then $f$ and $g$ possess a common fixed point. In particular, W. Boyce $[1$, Corollary 5] has shown that it suffices to assume the family $\left\{g^{n}: n \in \boldsymbol{N}\right\}$, iterates of $g$, is equicontinuous on $I$. This result has been extended by J. Cano [2, Theorems 1 and 2] who has required that either $g$ has a closed interval for its set of fixed points $F(g)$, or $F(g)$ coincides with $P(g)$, the set of all periodic points of $g$. It is worth underlining here that Corollary $5[1]$ as well as Theorem $1[2]$ give only sufficient conditions for the existence of a common fixed point of $f$ and $g$.

On the other hand, recently, Gerald Jungck [7, Theorem 3.6] established the following interesting equivalence: a continuous self-map $g$ of $I$ has a common fixed point with every continuous map $f: I \mapsto I$ that nontrivially commutes with $g$ on the set of coincidence points of $f$ and $g$ if and only if $P(g)=F(g)$.

Our purpose here is to give other necessary and sufficient criteria of this type (see Theorems 1, 2 and 3). We also obtain a variant of Jungck's Theorem in more abstract settings as compact and convex subsets of a normed linear space (see Proposition 1).

\section{EQUiVALENT CONDITIONS}

Following Boyce [1] and Cano [2] we define the classes of maps:

$$
\begin{aligned}
\boldsymbol{B} \doteq\left\{g: I \mapsto I \mid\left\{g^{n}: n \in \boldsymbol{N}\right\} \text { is equicontinuous on } I\right\}, \\
\boldsymbol{C}_{1} \doteq\{g: I \mapsto I \mid g \text { is continuous and } F(g) \text { is a closed interval }\}, \\
\boldsymbol{C}_{2} \doteq\{g: I \mapsto I \mid g \text { is continuous and } F(g)=P(g)\} .
\end{aligned}
$$

Received by the editors September 9, 1993 and, in revised form, March 29, 1995.

1991 Mathematics Subject Classification. Primary 54H25; Secondary 54E45.

Key words and phrases. Common fixed points, equicontinuity, commuting maps, iterates, unit interval. 
Let $f$ and $g$ be continuous self-maps of $I$. Then we have :

(1) If $f$ and $g$ commute on $I$ and $g \in B$ then $F(f) \cap F(g) \neq \emptyset$ [1, Corollary 5].

(2) If $f$ and $g$ commute on $I$ and $g \in C_{1} \cup \boldsymbol{C}_{2}$ then $F(f) \cap F(g) \neq \emptyset$; moreover, $B \subseteq \boldsymbol{C}_{1}$. If $g \in B$ and $F(g)$ is not a singleton then $g \in C_{2}$ [2, Theorems 1 and 2].

Next, by [7, Theorem 3.6], $g \in \boldsymbol{C}_{2}$ if and only if $F(f) \cap F(g) \neq \emptyset$ for every continuous map $f: I \mapsto I$ such that the set of coincidence points of $f$ and $g$ is non-empty, and $f$ and $g$ commute on it.

Inspired by the last result we give other characterizations of the classes $\boldsymbol{C}_{1}, \boldsymbol{C}_{2}$ and $\boldsymbol{B}$.

Theorem 1. Let $g$ be a continuous self-map of I. Then the following conditions are equivalent:

(i) $g \in C_{1}$;

(ii) the family $\left\{g^{n}: n \in N\right\}$ is equicontinuous on $F(g)$, or $F(g)$ is a singleton;

(iii) $g$ has a common fixed point with every continuous map $f: I \mapsto I$ that commutes with $g$ on $F(g)$.

Proof. (i) $\Rightarrow$ (ii). If $F(g)$ is a singleton, we are done. So suppose $F(g)=[a, b]$ and $a<b$. Obviously, it suffices to show that $\left\{g^{n}: n \in \boldsymbol{N}\right\}$ is equicontinuous at the points $a$ and $b$. And because of symmetry, we consider only the point $a$. The case when $a=0$ is trivial. So suppose $a>0$. Fix an $\epsilon \in(0, b-a)$. By the continuity, there exists a $\delta \in(0, \epsilon)$ such that

$$
a-\epsilon<g(x)<a+\epsilon \text { for all } x \in(a-\delta, a) \cap I .
$$

We shall apply induction on $n$ to show that, for all $n \in N$,

$$
a-\epsilon<g^{n}(x)<a+\epsilon \text { for all } x \in(a-\delta, a) \cap I .
$$

By (1), (2) holds if $n=1$. Assuming (2) holds for $n=1,2, \ldots, k$, we shall prove it for $k+1$. Fix an $x \in(a-\delta, a) \cap I$. If $a \leq g^{k}(x)<a+\epsilon$ then $g^{k}(x) \in F(g)$ since $a+\epsilon<b$ so (2) is fulfilled for $n=k+1$. Assume now that $g^{k}(x)<a$. Then $g^{i}(x)<a$ for $i=1,2, \ldots, k$; for otherwise, by induction hypothesis, $a \leq g^{i}(x)<a+\epsilon$ for some $i, 1 \leq i \leq k$ so $g^{i}(x) \in F(g)$, which implies $g^{k}(x) \in F(g)$ and hence $g^{k}(x) \geq a$, a contradiction. Since $F(g)=[a, b]$, we have $g(y)>y$ for all $y \in[0, a)$. In particular, $g^{i}(x)>g^{i-1}(x)$ for $i=1,2, \ldots, k$, which implies $g^{k}(x)>x$. Since $x>a-\delta$ and $g^{k}(x)<a$, we obtain that $g^{k}(x) \in(a-\delta, a) \cap I$. By $(1), a-\epsilon<g^{k+1}(x)<a+\epsilon$, which completes the induction.

Since $\delta<\epsilon<b-a$, we have $g^{n}(x)=x$ for $x \in[a, a+\delta)$ and $n \in N$. So finally, $a-\epsilon<g^{n}(x)<a+\epsilon$ for all $x \in(a-\delta, a+\delta)$ and $n \in \boldsymbol{N}$. This proves $\left\{g^{n}: n \in \boldsymbol{N}\right\}$ is equicontinuous at the point $a$.

(ii) $\Rightarrow$ (i). This implication follows from the proof of Cano's Theorem 1 [2].

(i) $\Rightarrow$ (iii). If $f$ commutes with $g$ on $F(g)$ then $F(g)$ is $f$-invariant so $\left.f\right|_{F(g)}$ has a fixed point since $F(g)$ is a closed interval.

(iii) $\Rightarrow$ (i). Suppose $F(g)$ is not an interval. There exist $a, b \in F(g), a<b$, such that $(a, b) \cap F(g)=\emptyset$. Define the map $f: f(x) \doteq b$ for $x \in[0, a], f(x) \doteq$ $-x+a+b$ for $x \in(a, b)$, and $f(x) \doteq a$ for $x \in[b, 1]$. Then $f$ is continuous and for $x \in F(g)$, either $x \in[0, a]$ and then $f(g(x))=g(f(x))=b$, or $x \in[b, 1]$ and then $f(g(x))=g(f(x))=a$. Thus $f$ and $g$ commute on $F(g)$, but $F(f) \cap F(g)=\emptyset$, which contradicts (iii). 
The following example shows that we cannot omit the condition " $F(g)$ is a singleton" in (ii) of Theorem 1.

Example 1. Define the map $g$ on $I$ as follows:

$$
g(x) \doteq 1 \text { for } x \in\left[0, \frac{1}{4}\right], g(x) \doteq-2 x+\frac{3}{2} \text { for } x \in\left(\frac{1}{4}, \frac{3}{4}\right), g(x) \doteq 0 \text { for } x \in\left[\frac{3}{4}, 1\right] .
$$

Clearly, $F(g)=\left\{\frac{1}{2}\right\}$ so $g \in \boldsymbol{C}_{1}$. However, it is easy to verify that the family $\left\{g^{n}: n \in N\right\}$ is not equicontinuous at the point $\frac{1}{2}$.

Theorem 2. Let $g$ be a continuous self-map of $I$. Then the following conditions are equivalent:

(i) $g \in \boldsymbol{C}_{2}$;

(ii) the sequence $\left\{g^{n}\right\}_{n=1}^{\infty}$ is pointwise convergent on $I$;

(iii) $g$ has a common fixed point with every continuous map $f: I \mapsto I$ that commutes with $g$ on $F(f)$.

Proof. That (i) implies (ii) was proved by S. C. Chu and R. D. Moyer [3, Theorem 1] and, independently, by E. M. Coven and G. A. Hedlund [4, Theorem 2]. To prove (ii) implies (iii) fix an $x \in F(f)$. Since, by the commutativity, $F(f)$ is $g$-invariant we have $g^{n}(x) \in F(f)$ for $n \in N$. By (ii), $\left\{g^{n}(x)\right\}_{n=1}^{\infty}$ converges to some $z \in I$. Then $z \in F(f) \cap F(g)$ since $F(f)$ is closed and $g$ is continuous. To prove (iii) implies (i) it suffices to show that for any non-empty closed $g$-invariant set $C \subseteq I$, $C \cap F(g) \neq \emptyset$ and then apply [3, Theorem 1]. Fix such a set $C$. There exists a continuous map $f: I \mapsto I$ such that $F(f)=C$. If $x \in F(f)$ then $g(f(x))=g(x)$ and $f(g(x))=g(x)$ since $C$ is $g$-invariant. Thus $f$ and $g$ commute on $F(f)$ so, by (iii), $F(f) \cap F(g) \neq \emptyset$, i.e., $C \cap F(g) \neq \emptyset$.

Remark 1. The sufficiency part of Jungck's Theorem 3.6 [7] is easily subsumed by Theorem 2: if $f$ and $g$ commute at their coincidence points, $P(g)=F(g)$ and $f(a)=g(a)$ then $f^{n}(a)=g^{n}(a)$ for $n \in N$. By Theorem $2((i) \Rightarrow($ ii $)),\left\{g^{n}(a)\right\}_{n=1}^{\infty}$ is convergent to some $b$. Then, by the continuity, $b$ is a common fixed point of $f$ and $g$.

Before stating the next theorem let us notice that a common fixed point theorem for a family of commuting maps would be trivial if we assumed one of them had a unique fixed point. This justifies a use of the assumption " $F(g)$ is not a singleton" in Theorem 3 below.

Theorem 3. Let $g$ be a continuous self-map of I such that $F(g)$ is not a singleton. Then the following conditions are equivalent:

(i) $g \in B$;

(ii) the sequence $\left\{g^{n}\right\}_{n=1}^{\infty}$ is uniformly convergent on $I$;

(iii) $g$ has a common fixed point with every continuous map $f: I \mapsto I$ that commutes with $g$ either on $F(f)$, or on $F(g)$.

Proof. (i) $\Rightarrow$ (ii). If $g \in \boldsymbol{B}$ and $F(g)$ is not a singleton then, by [2, Theorem 2], $g \in \boldsymbol{C}_{2}$. By Theorem 2, $\left\{g^{n}\right\}_{n=1}^{\infty}$ is pointwise convergent on $I$ which implies (ii), since $\left\{g^{n}: n \in N\right\}$ is equicontinuous.

(ii) $\Rightarrow$ (iii). This implication easily follows from Theorems 1 and 2 .

(iii) $\Rightarrow$ (i). By Theorem 2, $\left\{g^{n}\right\}_{n=1}^{\infty}$ is pointwise convergent, which implies $F(g)=$ $F\left(g^{2}\right)$. On the other hand, by Theorem $1, F(g)$ is an interval. Therefore, $F\left(g^{2}\right)$ is 
an interval, so by [1, Lemma 1 and Theorem 5], $\left\{g^{n}: n \in N\right\}$ is equicontinuous, i.e., $g \in B$.

Remark 2. The condition that $F(g)$ is not a singleton is necessary in Theorem 3 (consider the map $g(x) \doteq 1-x(x \in I)$, for which (i) holds but (ii) is not fulfilled). Further, Example 1 shows that one cannot modify Theorem 3 similarly to Theorem 1. More precisely, we cannot add in (ii) of Theorem 3 the text "or $F(g)$ is a singleton" and, simultaneously, remove the assumption " $F(g)$ is not a singleton" occurring at the beginning of Theorem 3.

We now give a partial extension of Theorem 2 for maps on subsets of a normed linear space.

Proposition 1. Let $A$ be a non-empty compact and convex subset of a normed linear space and let $g$ be a continuous self-map of $A$. Then the following conditions are equivalent:

(i) for any non-empty closed and g-invariant set $C \subseteq A, C \cap F(g) \neq \emptyset$;

(ii) $g$ has a common fixed point with every continuous map $f: A \mapsto A$ that commutes with $g$ on $F(f)$.

Remark 3. It follows from [3, Theorem 1] that in case when $A=I$, the conditions (i) of Proposition 1 and (i) of Theorem 2 are equivalent.

Proof of Proposition 1. (i) $\Rightarrow$ (ii). Let a continuous map $f: A \mapsto A$ commute with $g$ on $F(f)$. Then $F(f)$ is $g$-invariant and closed. Moreover, by Schauder's Fixed Point Theorem, $F(f)$ is non-empty. So by (i), we get $F(f) \cap F(g) \neq \emptyset$.

(ii) $\Rightarrow$ (i). Let $C$ be a non-empty closed $g$-invariant subset of $A$. We show that $C \cap F(g) \neq \emptyset$. The case when $C=A$ is trivial. So assume $C \neq A$ and fix a point $a \in A \backslash C$. There exists a continuous function $\phi: A \mapsto I$ such that $\phi^{-1}(0)=a$ and $\phi^{-1}(1)=C$ (see, e.g., [5, Theorem 1.5.19, p.69]). Assume further that $0 \in C$. Define a map $f$ by $f(x) \doteq \phi(x) x$ for $x \in A$. Then $f(A) \subseteq A$ by convexity, and $F(f)=C$, since $x=f(x)$ iff $x=0$ or $\phi(x)=1$. For $x \in C, g(f(x))=g(x)$ since $f(x)=x$, and $f(g(x))=g(x)$ since $C$ is $g$-invariant and $F(f)=C$. Thus $f$ and $g$ commute on $F(f)$. By (ii), $F(f) \cap F(g) \neq \emptyset$, i.e., $C \cap F(g) \neq \emptyset$. In case when $0 \notin C$, fix a point $c \in C$, consider the sets $A^{\prime} \doteq A-c, C^{\prime} \doteq C-c$, and repeat the above argument to deduce there exists a continuous map $f^{\prime}: A^{\prime} \mapsto A^{\prime}$ such that $F\left(f^{\prime}\right)=C^{\prime}$. Next, define a map $f$ as $f(x) \doteq f^{\prime}(x-c)+c$ for $x \in A$ and apply (ii) for such a map $f$ to obtain that $C \cap F(g) \neq \emptyset$.

\section{ACKNOWLEDGEMENT}

The author is grateful to the referee for suggesting some stylistic and expositional changes of the text of this paper.

\section{REFERENCES}

[1] W. M. Boyce, Г-compact maps on an interval and fixed points, Trans. Amer. Math. Soc. 160 (1971), 87-102. MR 43:6374

[2] J. CANO, Common fixed points for a class of commuting mappings on an interval, Proc. Amer. Math. Soc. 86 (1982), 336-338. MR 83h:54054

[3] S. C. ChU and R. D. Moyer, On continuous functions, commuting functions, and fixed points, Fund. Math. 59 (1966), 90-95. MR 34:805

[4] E. M. Coven and G. A. Hedlund, Continuous maps of the interval whose periodic points form a closed set, Proc. Amer. Math. Soc. 79 (1980), 127-133. MR 81a:54042 
[5] R. Engelking, General topology, Polish Scientific Publishers, Warszawa, 1977. MR 58:18316b

[6] J. P. Huneke, Two counterexamples to a conjecture on commuting continuous functions of the closed unit interval, Abstract no. 67T-2311, Notices Amer. Math. Soc. 14 (1967), 284.

[7] G. JungCK, Common fixed points for compatible maps on the unit interval, Proc. Amer. Math. Soc. 115 (1992), 495-499. MR 92m:54076

Institute of Mathematics, Technical University of Łódź, Żwirki 36, 90-924 Łódź, POLAND

E-mail address: jachymsk@lodz1.p.lodz.pl 